\title{
Perspective Piece \\ Expanding Opportunities for American Descendants of Slavery to Build a More Inclusive and Diverse Global Health Workforce
}

\author{
Tishina Okegbe* \\ Social Solutions International, Inc., Rockville, Maryland
}

\begin{abstract}
Historically, the terms African American and Black have been used interchangeably to describe any person with African ancestry living in the United States. However, Black Americans are not a monolith, and legitimate differences exist between those with generational roots in the United States and either African or Caribbean immigrants. American descendants of slavery (ADOS) are underrepresented in many fields, but I have noticed during my decades long career in global health that they are acutely absent in this field. Here, I offer seven recommendations to improve recruitment, retention, and advancement of ADOS in the global health field. Immediate implementation of these recommendations will not only bring diverse perspectives and immense capacity to the field but also allow ADOS an opportunity to engage in compelling and meaningful work and to collaborate with those from their ancestral homelands.
\end{abstract}

Historically, the terms African American and Black have been used interchangeably to describe any person with African ancestry living in the United States. However, although individuals with melanated skin are often perceived as Black Americans in our society, Black Americans are an extremely heterogeneous population. In 2016, a new phrase entered our collective lexicon to capture some of this nuance, categorizing Black Americans with generational roots in the United States as American descendants of slavery (ADOS), distinct from African or Caribbean immigrants. Although still a somewhat controversial idea, it is important to recognize that legitimate differences exist between these groups.

Although ADOS are underrepresented in many career fields, I have noticed throughout my decade-long career that compared with either African and Caribbean immigrants or firstgeneration Black Americans (i.e., native-born citizens who have one or more foreign born parents, like myself), ADOS are especially underrepresented in the American global health workforce. $\dagger$ The differences in lived experience between ADOS and African and Caribbean immigrants are valuable and should be celebrated, but it is time to recognize the important and beneficial perspective that ADOS can offer to the global health field.

Although I would love to supply data to supplement my lived experience, the extant data do not offer a designation to distinguish among ADOS, African or Caribbean immigrants, or first-generation Black Americans. Rather, this population is typically lumped together as Black or African American. Furthermore, many U.S. program implementers and policymakers do not share staff workforce demographic data broken down by racial and/or ethnic groups, if such data are

*Address correspondence to Tishina Okegbe, Social Solutions International, Inc., 6153 Executive Boulevard, Rockville, MD 20852. E-mail: tokegbe@socialsolutions.biz

† Herein defined as U.S.-based program implementers, policymakers, and researchers who identify, develop, and execute global health efforts worldwide, which includes professionals who work for nongovernmental organizations, private sector organizations, educational institutions, and U.S. government agencies. collected at all. This remains a critical gap as we try to better understand the racial and ethnic composition of the American global health workforce.

At a critical moment when our world, and particularly the United States, is undergoing a racial reckoning and is grappling with anti-Black sentiment, the time is now ripe to capitalize on the momentum and advocate for the inclusion of more diverse voices at the global health table. Intentional effort must be undertaken to attract ADOS to global health careers, and toward that end, I offer the following recommendations.

\section{CONDUCT OUTREACH TO MIDDLE-SCHOOL AND HIGH-SCHOOL STUDENTS}

American students, including ADOS, grow up with a very U.S.-centric worldview. During elementary and high school, students are often not aware of potential career opportunities outside of traditional paths, and a career in international relations is rarely considered as an option. Exposure to global health career options at a young age is critical to peak interest and curiosity in the field and ensure that students have opportunities to pursue relevant trainings and experiences. This intentional outreach will create a pipeline of ADOS students from varied backgrounds and facilitate a more diverse workforce for the next generation of global health workers. The science, technology, engineering, and mathematics (STEM) community has implemented this outreach strategy quite well over the last few decades, targeting young people from traditionally underrepresented backgrounds and providing opportunities to explore STEM fields, ranging from educational programs and science fairs to hands-on learning experiences at universities. ${ }^{1}$

PROVIDE PAID INTERNSHIP OPPORTUNITIES FOR ADOS UNDERGRADUATE AND GRADUATE STUDENTS WITHOUT THE REQUIREMENT OF PREVIOUS TRAVEL EXPERIENCE

Global health is unusual among professions as international travel necessarily comprises a large component of the work. In reviewing candidates for global health positions, previous 
overseas work or volunteer experience is weighted heavily. The consideration of previous overseas experience may be a limiting factor for individuals from historically underrepresented backgrounds and could preclude otherwise excellent candidates from securing opportunities. It is also critical to provide paid internship opportunities as ADOS individuals are less likely than others to have the means to support themselves given the U.S. racial wealth gap.

\section{CONDUCT REDACTED RECRUITMENT, EMPLOY DIVERSE INTERVIEW PANELS, AND STANDARDIZE INTERVIEWS}

Data show that bias generally exists during the recruitment process, and that such bias can be mitigated by redacting distinguishing characteristics such as a candidate's name, university of matriculation, and age during the initial screening process. $^{2}$ The adoption of such strategies and requiring diversity, equity, and inclusion training for panel participants can limit a recruiter's unconscious bias and allow an ADOS candidate to advance to the next round of consideration. It is important to note, however, that there are caveats with this approach, as removing this information might limit a recruiter's ability to fully contextualize the candidate's attributes. It is also important to include diverse representation on the hiring panel to mitigate unconscious bias and ensure that perspectives from underrepresented racial and/or ethnic groups are considered and valued. This also serves to model an inclusive workplace for the applicant.

\section{RECRUIT STUDENTS FROM HISTORICALLY BLACK COLLEGES AND UNIVERSITIES (HBCUS).}

Historically Black colleges and universities offer a highly qualified and educated source of talent for global health careers. These institutions, founded out of necessity during segregation, served as a refuge for African American education, and now educate roughly $9 \%$ of Black students enrolled in postsecondary education. Given that HBCUs produce a significant number of the Black STEM professionals who complete bachelor's degrees annually, conducting career and internship fairs on HBCU campuses across the country could introduce global health careers to ADOS students and expose them to opportunities that they may have been unaware of. ${ }^{3}$ Similar efforts have been made for STEM professions, and large global organizations, such as Google and Lockheed Martin, have developed recruitment initiatives geared specifically toward HBCUs.

\section{PRIORITIZE PROFESSIONAL DEVELOPMENT OPPORTUNITIES FOR ADOS}

Successfully recruiting and onboarding ADOS candidates is just the first step. Retaining ADOS employees and offering a clear path for advancement is equally if not more important, as Black staff are often overrepresented in internships and entry-level positions, but underrepresented in leadership positions. ${ }^{4}$ To this end, it is critical that ADOS employees have access to professional development opportunities, including mentorship, stretch assignments designed to challenge employees to grow beyond their current skillset, and leadership roles to hone the skills needed to advance their careers.

\section{LISTEN TO AMERICAN DESCENDANTS OF SLAVERY EMPLOYEES, AND HEAR THEIR LIVED EXPERIENCES}

It is important to foster a warm and inviting workplace culture that allows ADOS individuals to "show up" as their authentic selves. It is critical that topics of race are discussed openly and that ADOS employees feel comfortable speaking up or sharing their concerns. It is incumbent upon other colleagues to truly listen to and reflect on the concerns shared, and to commit to better understanding that perspective. This not only involves empathy but may also require colleagues to do outside reading and learning to gain insight as to how living in the United States frames the work, experiences, and motivations of ADOS colleagues. The Internet contains a wealth of free resources, including curated reading lists, which may serve as a helpful starting point. Organizations such as PopWorks Africa also offer paid content and curriculum, such as the popular "Decolonizing Development" webinar, which is especially relevant for global health professionals.

\section{OFFER MANDATORY DIVERSITY, EQUITY, AND INCLUSION (DEI) ONBOARDING TRAINING AND REQUIRED ANNUAL REFRESHERS AT GLOBAL HEALTH ORGANIZATIONS}

Mandating DEI training for all employees can signal a firm organizational commitment to increasing awareness of systemic racial inequities to foster a more welcoming environment. Successful conversations around DEI should promote a greater recognition of unconscious bias and prejudice, and how they have manifested in the workplace, as well as offer opportunities for growth in these areas. It is also important that the trainings are carried out in a thoughtful way to prevent defensiveness and backlash from participants.

\section{CONCLUSION}

Although these recommendations are by no means an exhaustive list, each provides a concrete path to increase the number of ADOS entering the global health workforce and advancing into global health leadership. As more ADOS enter the global health field, it will also be critical to develop transparent data systems to accurately capture their presence and contributions, including disaggregated Black subpopulation metrics. ADOS bring a unique perspective and immense capacity, and a career in global health offers ADOS the opportunity to engage in compelling, meaningful work and an avenue to give back to and work in partnership with those from their ancestral homelands. American descendants of slavery engagement in global health will advance global health goals, and thus requires our careful attention and immediate, concrete action.

Furthermore, as ADOS participation in global health increases, they may also contribute significantly to ongoing conversations as the field examines and rectifies its colonial legacy. This is not to say that ADOS should play the role of mediator to address the ills of the past and present; however, their voices could offer new perspectives and ideas, challenging the status quo and catalyzing deep reflection and real action. As a recent Lancet article declares, "ours is a discipline that holds within itself a deep contradiction-global health 
was birthed in supremacy, but its mission is to reduce or eliminate inequities globally." 5 It is incumbent upon those in U.S. global health leadership, which is predominately white and male, to not only diversify the field domestically by welcoming in more ADOS but also actively nurture, recruit, and elevate talent from the Global South.

Received December 29, 2020. Accepted for publication January 28, 2021.

Published online March 17, 2021.

Author's address: Tishina Okegbe, Social Solutions International, Inc., Rockville, MD, E-mail: tokegbe@socialsolutions.biz.

This is an open-access article distributed under the terms of the Creative Commons Attribution (CC-BY) License, which permits unrestricted use, distribution, and reproduction in any medium, provided the original author and source are credited.

\section{REFERENCES}

1. Metz C, Downes S, Metz M, 2018. The in's and out's of science outreach: assessment of an engaging new program. Adv Physiol Educ 42: 487-492.

2. Bertrand M, Mullainathan S, 2003. Are Emily and Greg more employable than Lakisha and Jamal? A field experiment on labor market discrimination. Am Econ Rev 94: 991-1013.

3. Harper C, 2003. HBCUs, Black women, and STEM success. Higher Education Today. Available at: https://www.higheredtoday.org/ 2018/05/14/hbcus-black-women-stem-success/. Accessed November 10, 2020.

4. U.S. Equal Employment Opportunity Commission, Office of Civil Rights and Diversity, USAID, 2020. Equal Employment Opportunity Program Status Report, Fiscal Year 2019, Form 71501. Washington, DC: USAID Office of Civil Rights and Diversity.

5. Abimbola S, Pai M, 2020. Will global health survive its decolonisation? Lancet 396: 1627-1628. 